\title{
A direct relation between confinement and chiral symmetry breaking in temporally odd-number lattice QCD
}

\section{Takahiro M. Doi*, Hideo Suganuma}

Department of Physics \& Division of Physics and Astronomy, Graduate School of Science, Kyoto University, Kitashirakawaoiwake, Sakyo, Kyoto 606-8502, Japan

E-mail: doi@ruby.scphys.kyoto-u.ac.jp

\section{Takumi Iritani}

High Energy Accelerator Research Organization (KEK), Tsukuba, Ibaraki 305-0801, Japan

In the lattice QCD formalism, we derive a gauge-invariant analytical relation connecting the Polyakov loop and the Dirac modes on a temporally odd-number lattice, where the temporal lattice size is odd, with the normal (nontwisted) periodic boundary condition. This analytical relation indicates that low-lying Dirac modes have little contribution to the Polyakov loop. Using lattice QCD simulations, we numerically confirm the analytical relation and the negligible contribution of low-lying Dirac modes to the Polyakov loop at the quenched level, i.e., the Polyakov loop is almost unchanged by removing low-lying Dirac-mode contribution from the QCD vacuum generated by lattice QCD in both confinement and deconfinement phases. Thus, we conclude that there is no one-to-one correspondence between confinement and chiral symmetry breaking in QCD. As a new method, modifying the Kogut-Susskind formalism, we develop a method for spin-diagonalizing the Dirac operator on the temporally odd-number lattice.

31st International Symposium on Lattice Field Theory - LATTICE 2013

July 29 - August 3, 2013

Mainz, Germany

\footnotetext{
* Speaker.
} 


\section{Introduction}

Color confinement and chiral symmetry breaking have been investigated as interesting nonperturbative phenomena in low-energy QCD in many analytical and numerical studies. However, their properties are not sufficiently understood directly from QCD. The Polyakov loop is an order parameter for quark confinement [1]. At the quenched level, the Polyakov loop is the exact order parameter for quark confinement, and its expectation value is zero in confinement phase and is nonzero in deconfinement phase. As for the chiral symmetry, low-lying Dirac modes are essential for chiral symmetry breaking in QCD, according to the Banks-Casher relation [2].

Not only the properties of confinement and chiral symmetry breaking in QCD but also their relation is an interesting challenging subject [3]. By removing QCD monopoles in the maximally Abelian gauge, both confinement and chiral symmetry breaking are lost in lattice QCD [4]. The transition temperatures of deconfinement and chiral restoration are almost same in finite temperature QCD [5]. From these facts, it is suggested that confinement and chiral symmetry breaking are strongly correlated. In recent lattice-QCD numerical studies, however, it is found that confinement properties do not change by removing low-lying Dirac modes from the QCD vacuum, which means no one-to-one correspondence between confinement and chiral symmetry breaking in QCD [6].

In this study, we derive an analytical relation connecting the Polyakov loop and the Dirac modes on a temporally odd-number lattice, and discuss the relation between confinement and chiral symmetry breaking. As a by-product, we develop a new method for spin-diagonalizing the Dirac operator on the temporally odd-number lattice by modifying the Kogut-Susskind (KS) formalism. Using this method, we numerically confirm the analytical relation.

\section{Dirac modes in lattice QCD}

In this section, we review the Dirac operator, its eigenvalues and its eigenmodes (Dirac modes) in $\mathrm{SU}\left(N_{\mathrm{c}}\right)$ lattice QCD [6]. We use a standard square lattice with spacing $a$, and the notation of sites $s=\left(s_{1}, s_{2}, s_{3}, s_{4}\right)\left(s_{\mu}=1,2, \cdots, N_{\mu}\right)$, and link-variables $U_{\mu}(s)=\mathrm{e}^{i a g A_{\mu}(s)}$ with gauge fields $A_{\mu}(s) \in s u\left(N_{c}\right)$ and gauge coupling $g$. In lattice QCD, the Dirac operator $\not D=\gamma_{\mu} D_{\mu}$ is given by

$$
\not D_{s, s^{\prime}}=\frac{1}{2 a} \sum_{\mu=1}^{4} \gamma_{\mu}\left[U_{\mu}(s) \delta_{s+\hat{\mu}, s^{\prime}}-U_{-\mu}(s) \delta_{s-\hat{\mu}, s^{\prime}}\right]
$$

with $U_{-\mu}(s) \equiv U_{\mu}^{\dagger}(s-\hat{\mu})$. Here, $\hat{\mu}$ is the unit vector in direction $\mu$ in the lattice unit. In this paper, we define all the $\gamma$-matrices to be hermite as $\gamma_{\mu}^{\dagger}=\gamma_{\mu}$. Since the Dirac operator is anti-hermite in this definition of $\gamma_{\mu}$, the Dirac eigenvalue equation is expressed as

$$
\not D|n\rangle=i \lambda_{n}|n\rangle
$$

with the Dirac eigenvalue $i \lambda_{n}\left(\lambda_{n} \in \mathbf{R}\right)$ and the Dirac eigenstate $|n\rangle$. Note that the chiral partner $\gamma_{5}|n\rangle$ is also an eigenstate with the eigenvalue $-i \lambda_{n}$. Using the Dirac eigenfunction $\psi_{n}(s) \equiv\langle s \mid n\rangle$, the explicit form for the Dirac eigenvalue equation is written by

$$
\frac{1}{2 a} \sum_{\mu=1}^{4} \gamma_{\mu}\left[U_{\mu}(s) \psi_{n}(s+\hat{\mu})-U_{-\mu}(s) \psi_{n}(s-\hat{\mu})\right]=i \lambda_{n} \psi_{n}(s)
$$




\section{Operator formalism in lattice QCD}

In this section, we present operator formalism in lattice QCD [6]. First, we define the linkvariable operator $\hat{U}_{ \pm \mu}$ by the matrix element,

$$
\left\langle s\left|\hat{U}_{ \pm \mu}\right| s^{\prime}\right\rangle=U_{ \pm \mu}(s) \delta_{s \pm \hat{\mu}, s^{\prime}} .
$$

Using the link-variable operator, the Polyakov loop $\left\langle L_{P}\right\rangle$ is expressed as

$$
\left\langle L_{P}\right\rangle=\frac{1}{3 V}\left\langle\operatorname{Tr}_{c}\left\{\hat{U}_{4}^{N_{4}}\right\}\right\rangle=\frac{1}{3 V}\left\langle\sum_{s} \operatorname{tr}_{c}\left\{U_{4}(s) U_{4}(s+\hat{t}) U_{4}(s+2 \hat{t}) \cdots U_{4}\left(s+\left(N_{4}-1\right) \hat{t}\right)\right\}\right\rangle,
$$

with the 4D lattice volume $V=N_{1} N_{2} N_{3} N_{4}$. Here, " $\operatorname{Tr}_{c}$ " denotes the functional trace of $\operatorname{Tr}_{c} \equiv \sum_{s} \operatorname{tr}_{c}$ with the trace $\operatorname{tr}_{c}$ over color index. In this formalism, the Dirac operator is simply expressed as

$$
\hat{\not D}=\frac{1}{2 a} \sum_{\mu=1}^{4} \gamma_{\mu}\left(\hat{U}_{\mu}-\hat{U}_{-\mu}\right)
$$

\section{A direct analytical relation between the Polyakov loop and Dirac modes in temporally odd-number lattice QCD}

We consider a temporally odd-number lattice, where the temporal lattice size $N_{4}$ is odd, with the normal (nontwisted) periodic boundary condition in both temporal and spatial directions. The spatial lattice size $N_{1 \sim 3}\left(>N_{4}\right)$ is taken to be even. As a key quantity, we first introduce

$$
I \equiv \operatorname{Tr}_{c, \gamma}\left(\hat{U}_{4} \hat{\not D}^{N_{4}-1}\right)
$$

with the functional trace $\operatorname{Tr}_{c, \gamma} \equiv \sum_{s} \operatorname{tr}_{c} \operatorname{tr} \gamma$ including also the trace $\operatorname{tr}_{\gamma}$ over spinor index. Its expectation value

$$
\langle I\rangle=\left\langle\operatorname{Tr}_{c, \gamma}\left(\hat{U}_{4} \hat{\not D}^{N_{4}-1}\right)\right\rangle
$$

is obtained as the gauge-configuration average in lattice QCD. In the case of large volume $V$, one can expect $\langle O\rangle \simeq \operatorname{Tr} O / \operatorname{Tr} 1$ for any operator at each gauge configuration.

From Eq. [3.3) $\hat{U}_{4} \hat{D}^{N_{4}-1}$ is expressed as a sum of products of $N_{4}$ link-variable operators. Since $N_{4}$ is odd, $\hat{U}_{4} \hat{\not}^{N_{4}-1}$ does not have any closed loops except for the term proportional to $\hat{U}_{4}^{N_{4}}$. Therefore, according to Elitzur's theorem and using Eq.(3.2), we obtain

$$
\langle I\rangle=\frac{1}{(2 a)^{N_{4}-1}}\left\langle\operatorname{Tr}_{c, \gamma}\left\{\hat{U}_{4}^{N_{4}}\right\}\right\rangle=\frac{4}{(2 a)^{N_{4}-1}}\left\langle\operatorname{Tr}_{c}\left\{\hat{U}_{4}^{N_{4}}\right\}\right\rangle=\frac{12 V}{(2 a)^{N_{4}-1}}\left\langle L_{P}\right\rangle .
$$

On the other hand, by performing the functional trace in Eq. (4.2) with the Dirac mode basis $|n\rangle$ satisfying $\sum_{n}|n\rangle\langle n|=1$, we find

$$
\langle I\rangle=\sum_{n}\left\langle n\left|\hat{U}_{4} \hat{D}^{N_{4}-1}\right| n\right\rangle=i^{N_{4}-1} \sum_{n} \lambda_{n}^{N_{4}-1}\left\langle n\left|\hat{U}_{4}\right| n\right\rangle .
$$

Combing Eqs. (4.3) and (4.4), we obtain the relation between the Polyakov loop $\left\langle L_{P}\right\rangle$ and the Dirac eigenvalues $i \lambda_{n}$ :

$$
\left\langle L_{P}\right\rangle=\frac{(2 a i)^{N_{4}-1}}{12 V} \sum_{n} \lambda_{n}^{N_{4}-1}\left\langle n\left|\hat{U}_{4}\right| n\right\rangle .
$$


This is a relation directly connecting the Polyakov loop and the Dirac modes, i.e., a Dirac spectral representation of the Polyakov loop, and is valid on the temporally odd-number lattice. From this relation 4.5, we can investigate each Dirac-mode contribution to the Polyakov loop individually.

From Eq.4.5], we can discuss the relation between confinement and chiral symmetry breaking in QCD. Because of the factor $\lambda_{n}^{N_{4}-1}$, the contribution from low-lying Dirac-modes with $\left|\lambda_{n}\right| \simeq 0$ is very small in the sum of RHS in Eq.4.5, compared to the other Dirac-mode contribution. In fact, the low-lying Dirac modes have little contribution to the Polyakov loop. This is consistent with the previous numerical lattice result that confinement properties are almost unchanged by removing low-lying Dirac modes from the QCD vacuum [6]. Thus, we conclude from the relation (4.5) that there is no one-to-one correspondence between confinement and chiral symmetry breaking.

\section{Modified KS formalism for temporally odd-number lattice}

The Dirac operator $\not D$ has a large dimension of $\left(4 \times N_{\mathrm{c}} \times V\right)^{2}$, so that the numerical cost for solving the Dirac eigenvalue equation is quite huge. This numerical cost can be partially reduced using the Kogut-Susskind (KS) formalism [1 6, 7. However, the original KS formalism can be applied only to the "even lattice" where all the lattice sizes $N_{\mu}$ are even number. In this section, we modify the KS formalism to be applicable to the odd-number lattice. Using the modified KS formalism, we can reduce the numerical cost in the case of the temporally odd-number lattice.

First, we recall the original KS formalism for even lattices. Using the matrix

$$
T(s) \equiv \gamma_{1}^{s_{1}} \gamma_{2}^{s_{2}} \gamma_{3}^{s_{3}} \gamma_{4}^{s_{4}}
$$

all the $\gamma$-matrices can be diagonalized as

$$
T^{\dagger}(s) \gamma_{\mu} T(s \pm \hat{\mu})=\eta_{\mu}(s) \mathbf{1}
$$

where $\eta_{\mu}(s)$ is the staggered phase,

$$
\eta_{1}(s) \equiv 1, \quad \eta_{\mu}(s) \equiv(-1)^{s_{1}+\cdots+s_{\mu-1}}(\mu \geq 2) .
$$

Then, one can spin-diagonalize the Dirac operator $\not D$ as

$$
\sum_{\mu} T^{\dagger}(s) \gamma_{\mu} D_{\mu} T(s+\hat{\mu})=\operatorname{diag}\left(\eta_{\mu} D_{\mu}, \eta_{\mu} D_{\mu}, \eta_{\mu} D_{\mu}, \eta_{\mu} D_{\mu}\right)
$$

where $\eta_{\mu} D_{\mu}$ is the KS Dirac operator given by

$$
\left(\eta_{\mu} D_{\mu}\right)_{s s^{\prime}}=\frac{1}{2 a} \sum_{\mu=1}^{4} \eta_{\mu}(s)\left[U_{\mu}(s) \delta_{s+\hat{\mu}, s^{\prime}}-U_{-\mu}(s) \delta_{s-\hat{\mu}, s^{\prime}}\right]
$$

Equation (5.4) shows fourfold degeneracy of the Dirac eigenvalue relating to the spiror structure, and then all the eigenvalues $i \lambda_{n}$ are obtained by solving the reduced Dirac eigenvalue equation

$$
\left.\left.\eta_{\mu} D_{\mu} \mid n\right)=i \lambda_{n} \mid n\right)
$$


Using the eigenfunction $\left.\chi_{n}(s) \equiv\langle s| n\right)$ of the KS Dirac operator, the explicit form of Eq.(5.6) reads

$$
\frac{1}{2 a} \sum_{\mu=1}^{4} \eta_{\mu}(x)\left[U_{\mu}(x) \chi_{n}(x+\hat{\mu})-U_{-\mu}(x) \chi_{n}(x-\hat{\mu})\right]=i \lambda_{n} \chi_{n}(x),
$$

where the relation between the Dirac eigenfunction $\psi_{n}(s)$ and the spinless eigenfunction $\chi_{n}(s)$ is

$$
\psi_{n}(s)=T(s) \chi_{n}(s) .
$$

Note here that the original KS formalism is applicable only to even lattices in the presence of the periodic boundary condition. In fact, the periodic boundary condition requires

$$
T\left(s+N_{\mu} \hat{\mu}\right)=T(s)(\mu=1,2,3,4),
$$

however, it is satisfied only on even lattices. Note also that, while the spatial boundary condition can be changed arbitrary, the temporal periodic boundary condition physically appears and cannot be changed at finite temperatures. Thus, the original KS formalism cannot be applied on the temporally odd-number lattice.

Now, we consider the temporally odd-number lattice, with all the spatial lattice size being even. Instead of the matrix $T(s)$, we introduce a new matrix

$$
M(s) \equiv \gamma_{1}^{s_{1}} \gamma_{2}^{s_{2}} \gamma_{3}^{s_{3}} \gamma_{4}^{s_{1}+s_{2}+s_{3}},
$$

where the exponent of $\gamma_{4}$ differs from $T(s)$ in Eq.5.1. As a remarkable feature, the requirement from the periodic boundary condition is satisfied on the temporally odd-number lattice:

$$
M\left(s+N_{\mu} \hat{\mu}\right)=M(s)(\mu=1,2,3,4) .
$$

Using the matrix $M(s)$, all the $\gamma$-matrices are transformed to be proportional to $\gamma_{4}$ :

$$
M^{\dagger}(s) \gamma_{\mu} M(s \pm \hat{\mu})=\eta_{\mu}(s) \gamma_{4}
$$

where $\eta_{\mu}(x)$ is the staggered phase given by Eq.(5.3). In the Dirac representation, $\gamma_{4}$ is diagonal as

$$
\gamma_{4}=\operatorname{diag}(1,1,-1,-1) \quad \text { (Dirac representation), }
$$

and we take the Dirac representation. Thus, we can spin-diagonalize the Dirac operator $\not D$ in the case of the temporally odd-number lattice:

$$
\sum_{\mu} M^{\dagger}(s) \gamma_{\mu} D_{\mu} M(s+\hat{\mu})=\operatorname{diag}\left(\eta_{\mu} D_{\mu}, \eta_{\mu} D_{\mu},-\eta_{\mu} D_{\mu},-\eta_{\mu} D_{\mu}\right),
$$

where $\eta_{\mu} D_{\mu}$ is the KS Dirac operator given by Eq. (5.5). Then, for each $\lambda_{n}$, two positive modes and two negative modes appear relating to the spinor structure on the temporally odd-number lattice. (Note also that the chiral partner $\gamma_{5}|n\rangle$ gives an eigenmode with the eigenvalue $-i \lambda_{n}$.) In any case, all the eigenvalues $i \lambda_{n}$ can be obtained by solving the reduced Dirac eigenvalue equation

$$
\left.\left.\eta_{\mu} D_{\mu} \mid n\right)= \pm i \lambda_{n} \mid n\right)
$$

just like the case of even lattices. The relation between the Dirac eigenfunction $\psi_{n}(s)$ and the sponless eigenfunction $\left.\chi_{n}(s) \equiv\langle s| n\right)$ is given by

$$
\psi_{n}(s)=M(s) \chi_{n}(s)
$$

on the temporally odd-number lattice. 


\section{Numerical confirmation for the relation between Polyakov loop and Dirac modes}

Using the modified KS formalism, Eq.(4.5) is rewritten as

$$
\left\langle L_{P}\right\rangle=\frac{(2 a i)^{N_{4}-1}}{3 V} \sum_{n} \lambda_{n}^{N_{4}-1}\left(n\left|\hat{U}_{4}\right| n\right) .
$$

Note that the (modified) KS formalism is an exact method for diagonalizing the Dirac operator and is not an approximation, so that Eqs. (4.5) and (6.1) are completely equivalent. In fact, the relation (4.5) can be confirmed by the numerical test of the relation 6.17.

We numerically calculate LHS and RHS of the relation 6.1), respectively, and compare them. We perform SU(3) lattice QCD Monte Carlo simulations with the standard plaquette action at the quenched level in both cases of confinement and deconfinement phases. For the confinement phase, we use $10^{3} \times 5$ lattice with $\beta \equiv 2 N_{\mathrm{c}} / g^{2}=5.6$ (i.e., $a \simeq 0.25 \mathrm{fm}$ ), corresponding to $T \equiv 1 /\left(N_{4} a\right) \simeq$ $160 \mathrm{MeV}$. For the deconfinement phase, we use $10^{3} \times 3$ lattice with $\beta=5.7$ (i.e., $a \simeq 0.20 \mathrm{fm}$ ), corresponding to $T \equiv 1 /\left(N_{4} a\right) \simeq 330 \mathrm{MeV}$. For each phase, we use 20 gauge configurations, which are taken every 500 sweeps after the thermalization of 5,000 sweeps.

As the numerical result, comparing LHS and RHS of the relation 6.17, we find that the relation 6.1) is almost exact for each gauge configuration in both confinement and deconfinement phases. Therefore, of course, the relation 6.1 is satisfied for the gauge-configuration average.

Next, we numerically confirm that the low-lying Dirac modes have negligible contribution to the Polyakov loop using Eq.6.11). By checking all the Dirac modes, we find that the matrix element $\left(n\left|\hat{U}_{4}\right| n\right)$ is generally nonzero. In fact, for low-lying Dirac modes, the factor $\lambda_{n}^{N_{4}-1}$ plays a crucial role in RHS of Eq. 6.1). Since RHS of Eq.6.1) is expressed as a sum of the Dirac-mode contribution, we calculate the Polyakov loop without low-lying Dirac-mode contribution as

$$
\left\langle L_{P}\right\rangle_{\mathrm{IR}-\mathrm{cut}} \equiv \frac{(2 i)^{N_{4}-1}}{3 V} \sum_{\left|\lambda_{n}\right|>\Lambda_{\mathrm{IR}}} \lambda_{n}^{N_{4}-1}\left(n\left|\hat{U}_{4}\right| n\right)
$$

with the infrared (IR) cut $\Lambda_{\mathrm{IR}}$ for the Dirac eigenvalue. The chiral condensate $\langle\bar{q} q\rangle$ is given by

$$
\langle\bar{q} q\rangle=-\frac{1}{V} \operatorname{Tr}_{c, \gamma} \frac{1}{\not D+m}=-\frac{1}{V} \sum_{n} \frac{1}{i \lambda_{n}+m}=-\frac{1}{V}\left(\sum_{\lambda_{n}>0} \frac{2 m}{\lambda_{n}^{2}+m^{2}}+\frac{v}{m}\right),
$$

where $m$ is the current quark mass and $v$ the total number of zero modes of $\not D$. The chiral condensate without the contribution from the low-lying Dirac-mode below IR cut $\Lambda_{\mathrm{IR}}$ is given by

$$
\langle\bar{q} q\rangle_{\Lambda_{\mathrm{IR}}}=-\frac{1}{V} \sum_{\lambda_{n}>\Lambda_{\mathrm{IR}}} \frac{2 m}{\lambda_{n}^{2}+m^{2}} .
$$

Here, we take the IR cut of $\Lambda_{\mathrm{IR}} \simeq 0.4 \mathrm{GeV}$. In the confined phase, this IR Dirac-mode cut leads to

$$
\frac{\langle\bar{q} q\rangle_{\Lambda_{\mathrm{R}}}}{\langle\bar{q} q\rangle} \simeq 0.02
$$

and almost chiral-symmetry restoration in the case of physical current-quark mass, $m \simeq 5 \mathrm{MeV}$.

We find that $\left\langle L_{P}\right\rangle \simeq\left\langle L_{P}\right\rangle_{\text {IR-cut }}$ is numerically satisfied for each gauge configuration in both confinement and deconfinement phases. Table 1 and 2 show a part of the numerical result on $\left\langle L_{P}\right\rangle$ and $\left\langle L_{P}\right\rangle_{\text {IR-cut }}$ for confinement and deconfinement phases, respectively. In this way, the Polyakov loop is almost unchanged by removing the contribution from the low-lying Dirac modes, which are essential for chiral symmetry breaking. From both analytical and numerical results, we conclude that there is no one-to-one correspondence between confinement and chiral symmetry breaking. 
Table 1: Numerical results for $\left\langle L_{P}\right\rangle$ and $\left\langle L_{P}\right\rangle_{\mathrm{IR}-\text { cut }}$ in lattice QCD with $10^{3} \times 5$ and $\beta=5.6$ for each gauge configuration, where the system is in confinement phase.

\begin{tabular}{|c|c|c|c|c|c|c|c|}
\hline configuration No. & 1 & 2 & 3 & 4 & 5 & 6 & 7 \\
\hline $\operatorname{Re}\left\langle L_{P}\right\rangle$ & 0.00961 & -0.00161 & 0.0139 & -0.00324 & 0.000689 & 0.00423 & -0.00807 \\
$\operatorname{Im}\left\langle L_{P}\right\rangle$ & -0.00322 & -0.00125 & -0.00438 & -0.00519 & -0.0101 & -0.0168 & -0.00265 \\
\hline $\operatorname{Re}\left\langle L_{P}\right\rangle_{\text {IR-cut }}$ & 0.00961 & -0.00160 & 0.0139 & -0.00325 & 0.000706 & 0.00422 & -0.00807 \\
$\operatorname{Im}\left\langle L_{P}\right\rangle_{\text {IR-cut }}$ & -0.00321 & -0.00125 & -0.00437 & -0.00520 & -0.0101 & -0.0168 & -0.00264 \\
\hline
\end{tabular}

Table 2: Numerical results for $\left\langle L_{P}\right\rangle$ and $\left\langle L_{P}\right\rangle_{\mathrm{IR}-\text { cut }}$ in lattice QCD with $10^{3} \times 3$ and $\beta=5.7$ for each gauge configuration, where the system is in deconfinement phase.

\begin{tabular}{|c|c|c|c|c|c|c|c|}
\hline configuration No. & 1 & 2 & 3 & 4 & 5 & 6 & 7 \\
\hline $\operatorname{Re}\left\langle L_{P}\right\rangle$ & 0.316 & 0.337 & 0.331 & 0.305 & 0.314 & 0.316 & 0.337 \\
$\operatorname{Im}\left\langle L_{P}\right\rangle$ & -0.00104 & -0.00597 & 0.00723 & -0.00334 & 0.00167 & 0.000120 & 0.0000482 \\
\hline $\operatorname{Re}\left\langle L_{P}\right\rangle_{\text {IR-cut }}$ & 0.319 & 0.340 & 0.334 & 0.307 & 0.317 & 0.319 & 0.340 \\
$\operatorname{Im}\left\langle L_{P}\right\rangle_{\text {IR-cut }}$ & -0.00103 & -0.00597 & 0.00724 & -0.00333 & 0.00167 & 0.000121 & 0.0000475 \\
\hline
\end{tabular}

\section{Summary and concluding remarks}

In this study, we have analytically derived a direct relation connecting the Polyakov loop and the Dirac modes in temporally odd-number lattice QCD, with the normal (nontwisted) periodic boundary condition. We have shown that the low-lying Dirac modes have little contribution to the Polyakov loop, which means no one-to-one correspondence between confinement and chiral symmetry breaking in QCD. As a new method, we have modified the KS formalism to perform the spin-diagonalizing of the Dirac operator on the temporally odd-number lattice. Using the modified KS formalism, we have numerically shown that the contribution of low-lying Dirac modes to the Polyakov loop is negligible in both confinement and deconfinement phases.

\section{Acknowledgements}

H.S. and T.I. are supported in part by the Grant for Scientific Research [(C) No.23540306, E01:21105006, No.21674002] from the Ministry of Education, Science and Technology of Japan. The lattice QCD calculation has been done on NEC-SX8R at Osaka University.

\section{References}

[1] H.J. Rothe, Lattice Gauge Theories, (World Scientific, 2012), and its references.

[2] T. Banks and A. Casher, Nucl. Phys. B169 (1980) 103.

[3] H. Suganuma, S. Sasaki and H. Toki, Nucl. Phys. B435 (1995) 207.

[4] O. Miyamura, Phys. Lett. $B 353$ (1995) 91; R.M. Woloshyn, Phys. Rev. D51 (1995) 6411.

[5] F. Karsch, Lect. Notes Phys. 583 (2002) 209, and its references.

[6] S. Gongyo, T. Iritani and H. Suganuma, Phys. Rev. D86 (2012) 03451; T. Iritani and H. Suganuma, [arXiv:1305.4049 [hep-lat]]; T. Iritani et al., PoS (Confinement X) (2013) 053.

[7] J.B. Kogut and L. Susskind, Phys. Rev. D11 (1975) 395. 